\title{
LOW BACK PAIN OF OBSCURE ORIGIN
}

\author{
By WALTER MERCER, M.B., F.R.C.S. (Edin.). \\ (Assistant Surgeon, Royal Infirmary, Edinburgh and Surgeon, Chalmers' Hospital, \\ Edinburgh, etc.)
}

Every branch of surgery has its problem, and surely none has a more perplexing one than the low back pain of orthopædics. At one time this problem, with which we were confronted daily, seemed a hopeless one; but gradually, with increased knowledge and after an immense amount of carefully directed research, order and hope have evolved from chaos, and a more exact diagnosis has been made possible, so that now there is hope for the great majority of low back cases seen by the specialist orthopædist. The evolution of the treatment of "backs" is interesting. There was an early period when little or no attempt was made to make an accurate diagnosis, and all backs were treated merely symptomatically. Shortly before the Great War the pendulum swung the other way and most cases were diagnosed as "sacro-iliac strain," so obscuring the essential problem. I believe that now an accurate diagnosis can be made in the great majority of cases so that, with a reasonable line of treatment ascertainable, the outlook is at least a hopeful one. There still remains, however, a group of cases in which the patho genesis is indefinite and in which derangement can only be assumed. These cases are difficult to assess, to treat, or to forecast as to the future, and it is to this group that I particularly refer here.

\section{THE HISTORY.}

In the management of a case of low back pain the history of the condition should be gone into carefully, and the examination of the back and of the patient generally should be as systematic and as meticulous as is humanly possible. Only by so doing can a differential diagnosis between the various causes of backache be made and a régime of treatment which is likely to be successful laid down. The best method undoubtedly is to have a questionnaire form made up, so that with each patient a definite order of examination is undertaken and no point of the examination is omitted. Great attention is paid to the patient's story, especially in regard to the mode of onset and the question of trauma and the type of trauma, the location and radiation of the pain, its frequency, duration, and factors which influence it. The patient's description of the distribution of the pain is helpful when we consider the innervation of the lumbo-sacral and sacro-iliac joints. In lumbo-sacral arthritis the pain is referred along the fifth lumbar and first sacral nerves, while in a sacro-iliac condition the pain is referred along the fourth and fifth lumbar and the first and second sacral nerves. In other words, while in the former condition the pain may be confined to the lower leg, the posterior aspect of the thigh is also complained of in a sacro-iliac disturbance.

\section{THE EXAMINATION.}

The examination should be just as careful, and inspection, palpation, motion and special tests are carried out in turn. Smith-Petersen has pointed out how 
important a part of the examination palpation is. He does this in a definite systematic routine in the following order (See Fig. I):-

“ (I) Posterior superior spine: used simply as a bony landmark. (2) Inferior sacro-iliac ligaments: sensitiveness in this region is in favour of a sacro-iliac condition. (3) Sacro-sciatic notch: at this point the superior gluteal nerve curves around as it passes forward between the gluteus medius and the gluteus minimus muscles. Sensitiveness in this region is almost a constant finding in sacro-iliac cases. (4) Sciatic nerve trunk, half way between the ischial tuberosity and the greater trochanter at the level of the gluteal fold. Because of referred pain along this nerve, we sometimes get sensitiveness of the sciatic nerve trunk; but since the sciatic nerve contains branches from the fourth and fifth lumbar as well as the first, second, and third sacral, sensitiveness of the sciatic nerve trunk is not helpful in differential diagnosis between the two regions. (5) Posterior superior spine again: purely as a bony landmark. (6) Mesial to and lateral to the posterior crest of the ilium. In other words, the origin of the ilio-lumbar ligaments and the ilio-costalis muscle, respectively, from the ilium. Sensitiveness in this region is distinctly in favour of a lumbo-sacral condition. (7) The crest of the ilium: purely as a bony landmark and guide to the level of the spinous process of the fourth lumbar. (8) Spinous processes and inter-spinous ligaments of the fourth and fifth lumbar and first sacral. Sensitiveness in this region again is in favour of a lumbo-sacral condition. (9) Dorsum of the sacrum and articular facets of the fifth lumbar and first sacral. Sensitiveness in this region may occur with sacro-iliac as well as lumbo-sacral conditions because of the nerve supply from the fifth lumbar and first and second sacral nerves; consequently, sensitiveness here is of no particular value in differential diagnosis."

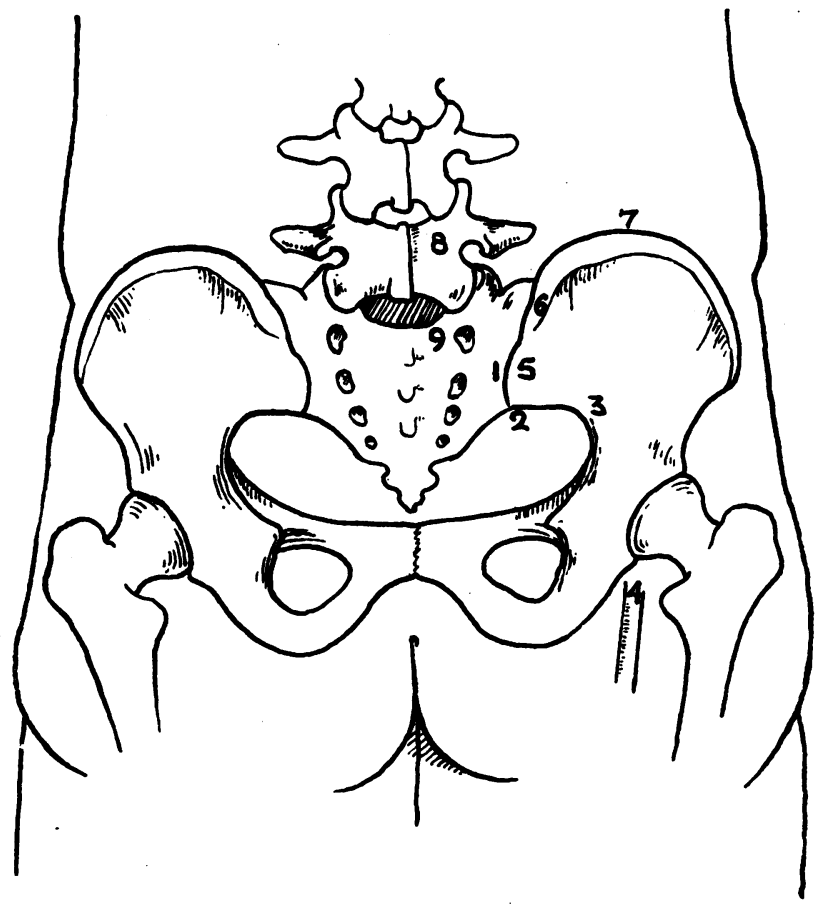

FIG. 1 .

The anatomical points concerned in the examination of a case of backache. (After Smith-Petersen.) 
Special tests are of great value in the differential diagnosis and location of low back pain. Forcible compression of the iliac crests may produce pain in strain of the sacro-iliac joint, but has no effect in a lumbo-sacral strain. Laguere's sign, $\stackrel{\vec{S}}{\rightarrow}$ which consists of forcing the leg in flexion, abduction and lateral rotation, causes 0 pain in an affected sacro-iliac joint, but has no effect upon a painful lumbo-sacral $\frac{}{\bar{N}}$ one. The straight-leg bending test of Goldthwaite, where the thigh is strongly flexed with the knee extended, produces a tension of the hamstring muscles and $\stackrel{D}{a}$ indirectly a rotatory force upon the painful sacro-iliac joint. This also produces is pain in sciatica, but sciatica will be diagnosed from a sacro-iliac arthritis by the $\overrightarrow{0}$ fact that the pain is definitely increased by dorsiflexing the foot in addition to the other manœuvre. In Gaenslen's sign, where movement is produced in the sacroiliac joint by flexing one extremity on the abdomen and hyperextending the $\bar{\square}$ opposite hip, pain is complained of at once in sacro-iliac conditions, owing to the 3 rotation of the affected joint. A rectal examination should be carried out in all $\vec{\omega}$ cases, but especially in inflammatory conditions of the sacro-iliac joint, since in these cases there is sufficient reaction, swelling and sensitiveness, to be detected per $\vec{f}$ rectum, particularly should the anterior sacro-iliac ligaments be affected. Tumour $\mathrm{W}_{\mathrm{N}}$ formation, either of the prostate gland or of the rectum itself, not infrequently produces in its early stages only low back pain.

\section{THE DIAGNOSIS.}

The first practical point in diagnosis is to determine:-

(I) whether the pain is a symptomatic one, referred from underlying $\vec{\theta}$ disease elsewhere, or

(2) whether the pain is due to a regional disturbance of the skeletat structures or the immediate surroundings.

\section{I.-Symptomatic Low Back Pain.}

Most of the cases of low back pain in the Orthopædic Department here are $\overrightarrow{\vec{O}}$ referred to us from the Gynæcological Department, and it is a very natural sequence that this should be so, for although it is widely held that most cases of back- $\vec{\sigma}$ ache in women are due to some menstrual disturbance, I believe that comparatively few of these cases can be attributed to pelvic disorders per se. A study of the nerve supply of the uterus draws attention to the fact that the innervation for the most part comes from the pelvic plexus, the inferior hypogastric plexus, the ovarian plexus, and only a few filaments from the sacral nerves. Careful examination usually shows that there are other more plausible causes for the production of this common complaint. Retroversion itself has little, if any, relation to backache, but it is 9 frequently found that after suspension of the uterus and repair of the markedly $\frac{D}{0}$ relaxed pelvic floor, a patient may gain considerable relief. The correction of the pelvic ptosis with automatic correction of the associated enteroptosis is probably the most important factor in giving relief. Backache is more prevalent in women $N$ than in men, especially in women who have borne children. The child-bearing function brings with it certain definite changes and distortions of the pelvic bones. The symphisis is separated during the period of gestation and delivery. The sacro-iliac synchondrosis spreads. The ligaments which retain the normal relationship of the articulation become relaxed. In certain circumstances these distortions ? remain uncorrected, and instability of the sacro-iliac joints results. In the last two 0 months of pregnancy women frequently complain bitterly of low back pain, which ${ }_{\mathbb{D}}$ is due to a greatly exaggerated lumbar lordosis produced by the forward and $\underset{\mathbb{D}}{\mathbb{Q}}$ 
downward pull of the pendulous abdomen. Support of the abdomen or recumbency are the methods of cure, but it is important for such cases to be cared for after the delivery. They should have massage and recumbent exercises to raise the muscular tone during the period in bed, and when allowed up adequate corset support to the abdomen is necessary. Sometimes such patients are permitted ten days after delivery to be up and about on the presumption that the relaxation that has taken place over a period of months has corrected itself. But we expect too much from nature. Nature cannot do everything and the help that we can give most easily is rest. It can readily be imagined what permanent changes must take place after eight or ten pregnancies in that number of years. Women who bear children, therefore, deserve special care and attention. A reasonable length of time must elapse before they are permitted to be up and about their daily routine. Then, and only then, will we avoid the disfiguring painful deformity so often seen in our comparatively young women. Only then will we see fewer of the women with wide hips and a pendulous abdomen- " the woman of 38 with the characteristic complaint and deformity of a grandmother of 6o." It is obvious, then, that the care of backache in women as well as in men is the responsibility of the orthopædist and not of the gynæcologist.

Referred backache from the gastro-intestinal tract is not infrequent. An overloaded colon may cause pain not only by its weight, but also by causing referred pain along the upper two lumbar nerves. Albee has been particularly impressed with the importance of gastro-intestinal toxæmia as an important factor in backache.

\section{2.--Regional Low Back Pain.}

One purposely omits from this discussion all fractures and dislocations of the spine, gross disease such as tuberculosis, and gross congenital anomalies such as spondylolisthesis.

From the analysis of many groups of statistics the causes of low back pain in order of relative importance are:-trauma, including sprains and strains, arthritis, and postural causes.

A. Trauma. Where trauma is the exciting factor the history is usually that of a mild wrench or sprain of the lower back suffered by one engaged in a hard labouring occupation. It is usually not sufficiently severe to make the patient stop work. The sequence thereafter is increasing discomfort in the lumbo-sacral region, continued over a long period of time, aggravated by work, relieved by rest, and without pain at night or morning stiffness.

In those cases where the sprain is of the joints-sacro-iliac or lumbo-sacralthe differentiation from chronic arthritis is very difficult, and most observers believe that the majority of such chronic cases are due to arthritis, and in this communication they are considered with the arthritic type.

Ligamentous and muscular sprains are produced by injuries similar to the above and are very common in the lumbar region. The pain is sudden, and though increased by certain movements, it is constantly felt during the acute stage, and only partly relieved by rest. In unilateral lesions it can be brought on by movements which demand stretching of the affected muscles or ligaments.

In addition to severe pain, muscular and ligamentous sprains of the lower back are often associated with limping and with muscular spasm, so that the 
patient holds his spine rigid and walks with a very slow and painful gait. It is usually almost impossible to decide whether the lesion is actually in the muscle or in a ligament, but when the muscle alone is affected, the symptoms are intensified when the muscle contracts and when it is put on the stretch. The underlying pathology is simple: there has usually been a rupture of some fibres, and the pain persists for a long time unless they get a chance to heal. Healing takes place through the medium of fibrous tissue, and if the muscle is continually subjected to slight strains during the process of repair, this fibrous tissue is apt to be excessive. In ligamentous injuries, the pain is deep-seated, and can be elicited both by movement of the spine and by pressure with the finger. In contrast to muscle injuries, active and passive movements in the same direction both produce pain.

Muscle or ligament strains, if of long standing, may be complicated by mild infective attacks, during which the pain becomes more diffuse and tenderness can be elicited over a wider area.

There are other traumatic conditions which we all see frequently following on a usual day's work - standing, stooping, washing, etc. We often overlook deformities of the lower extremities, especially those of the feet. Flat foot deformity means walking with the feet everted and changes in the pelvis. Other ætiological factors we can enumerate are various foci in the head (teeth, tonsils, sinuses), kidney and bladder involvements, and occasionally pelvic infection. Trauma plays no small part in the localization of these foci. The management of such cases does not call for the removal of tubes, ovaries or uterus, but demands a removal of the cause primarily, then an improvement of posture, change of habits, and rest.

B. Arthritis. Arthritis as a factor in low backache is found in the sacroiliac joint, the lumbo-sacral joint, and the smaller intervertebral joints. It is a senile degeneration, found most commonly in labouring men, caused by the long-continued stress of labour plus probably an element arising out of bad hygiene-poor mouth hygiene, incorrect kind and quantity of food, poor elimination, and possibly improper protection against the elements. These changes may cause no symptoms, except loss of motion, over long periods of time. In the great majority of cases it is an injury of some sort which brings to light the presence of hypertrophic changes in the spine. Apparently individuals can live and work for years in the presence of slowly developing osteo-arthritic changes without inconvenience. The principal symptoms are pain, stiffness, and some wasting of muscles. The pain is neuralgic and is relieved on movement. It is, in fact, worst after resting, and often better after exercise. The X-ray appearances are rarely characteristic in the early stages since bony changes are not then visible. The characteristic and later X-ray picture, however, is only a part of the disease. The soft tissue swelling about the calcareous deposit usually causes distressing tension and direct pressure upon the nerves.

If the history and symptomatology suggest arthritis, the probability is that either the sacro-iliac or the lumbo-sacral joint is the site of the main trouble. Arthritis is difficult to distinguish from a chronic sprain in either of those joints, except in the later stages when there is definite X-ray evidence, so that for practical purposes the two conditions may be considered together.

Sacro-iliac Strain. In the case of the sacro-iliac affection, although the symptoms vary considerably, certain features are constantly present. Pain is experienced over the posterior aspect of the joint, and may be elicited by digital pressure over that area. 
It is definitely increased by movements which put added strain on the joint, but not by pressure along the nerve root; it is constantly more severe at night because the recumbent position obliterates the normal lumbar lordosis and throws more strain on the joint. Exacerbations occur at the menstrual periods, since the increased congestion at that time causes softening of the joint ligaments, and increased joint mobility. Standing or sitting for long periods also leads to an aggravation of the pain. Lateral compression of the iliac crests may produce pain in the joint. Movements of the body on the thighs, or of the thighs on the body, are limited. Forward bending of the trunk with the knees held straight is restricted if the lesion is one of any magnitude. To determine whether this limitation is the result of spinal disease, or of sacro-iliac disease, the tension of the hamstring muscles should be released by allowing the patient to sit. Similar movements are now attempted: when the spine is involved, the limitation will be found to persist, but in lesions of the sacro-iliac joint flexion can now be carried out easily and without pain. When attempts are made to flex the hip, with the knee fully extended, marked limitation is present in sacro-iliac lesions, and attempts to increase the range of movement cause pain, referred either directly to the sacral region, or to the leg. This phenomenon is known as Goldthwaite's sign.

Albee states that the following symptom complex, when present, is almost pathognomonic of sacro-iliac disturbance-

(a) Pain in the region of the joint on turning over from the recumbent posture.

(b) Discomfort while lying on the back.

(c) Pain while sitting on a hard chair, relieved by sitting on the opposite buttock.

(d) Pain in the affected joint on forward bending.

(e) Pain on deep pressure over the affected joint.

(f) Listing of the whole spine to the opposite side.

(g) Positive Goldthwaite's sign.

The commonest feature in the X-ray picture of a sacro-iliac arthritis of moderate severity is the increased density along the margins of the joint, while irregularity of the joint line and proliferative changes at the inferior margin of the joint are not uncommon.

Differential Diagnosis. Sacro-iliac sprain differs from lumbago in that the latter is bilateral, while the pain is sharply stabbing in character, and is situated over the lumbar muscles, which are also tender on pressure. Goldthwaite's sign is absent.

Lumbo-Sacral Strain. The lumbo-sacral articulation is liable to be strained when leverage or trauma is applied from above with the spine in flexion. The pain and tenderness which result are situated at the lumbo-sacral junction, and the movements of the lumbar spine are restricted in all directions. A lumbo-sacral case will bend forward freely, however, whether sitting or standing, because he holds the lumbo-sacral region rigid and bends chiefly at the hip joints. Heavy, stout individuals, with a protruding abdomen, are most commonly affected. The hip-joint movements are usually free on both sides, but straight leg raising is occasionally limited in its last few degrees, when both legs are flexed. 
The pain is referred to the fifth lumbar and first sacral areas, and is therefore distributed to the lateral side of the leg, the dorsum of the foot, and sole of the foot. Tenderness is found over the ilio-lumbar ligament, the spinous processes of the fourth and fifth lumbar and first sacral vertebræ.

C. Postural Causes. Faulty attitudes may either cause or predispose to chronic strain. Many a case of postural backache has been cured in a gymnasium by corrective exercises for the weak musculature. Especially well do patients of the asthenic type respond to exercises. As a rule these individuals stand in a sloppy attitude of great muscular relaxation; the feet are pronated, the knees and hips flexed, the lumbar lordosis increased, the shoulders drooping and the abdomen protruding; and there is associated visceroptosis. These patients are actually held in the erect position by their ligaments, the weakened musculature showing loss of tone with resultant impoverished blood supply.

Certain occupations demand extreme use of the lumbo-sacral region, and those cases giving a history of the onset of backache some short time after excessive stooping, bending, lifting, or twisting motions, are classed as occupational. This group might be termed "chronic traumatic" in contra-distinction to that greater group- the acute traumatic type with immediate symptoms. This postural insufficiency is primarily in the muscles: a condition in which apparently normal musculature is not equal to the demand placed upon it. The commonest complaint is of an ache and a tired feeling. The ache at first is relieved by rest. Later ordinary rest is inadequate and the ache becomes constant. Further, if a muscle has been strained and is rested and then put into use too soon, the symptoms of soreness and stiffness are added. Muscles that are used in weightbearing when fatigued permit their load to be transmitted directly to the jointso around which they act, so straining and stretching the joint ligaments. The physical findings of muscular insufficiency vary somewhat according to the number of parts involved. There is muscle tenderness, tenderness over the ligamentous attachments, and deformities in the form of altered posture. During an acute attack there is muscle spasm in the lumbar area. In some cases there is a list to one side. Active motion is limited. Laguere's sign is positive and the sacro-iliac area is tender. The normal lumbar curve is obliterated. After the acute phase has subsided, the muscles relax and the tone seems below normal. The lumbar curve becomes accentuated to form a lordosis; the shoulders droop forward and the chest is depressed; the abdomen is prominent with a tendency to be pendulous. With the exception of the cases of long-standing which show osteoporosis, the X-ray findings are negative.

\section{TREATMENT.}

In all severe acute traumatic cases the patient is put to bed, sometimes with adhesive support round his low back area, but-oftener without it, because physiotherapy should be instituted as soon as possible. When there is much muscle spasm, head and pelvic traction is indicated. The traction is attached to both limbs with some 6 to Io pounds weight on each. Traction is particularly indicated in those cases with sciatic radiation and those with an asymmetrical posture, the weights being gradually increased as the patient can stand them until the posture is more normal. A small pillow is usually slipped under the lumbar area and gradually the normal lumbar curve is recovered. Local heat is applied to the back by means of an electric pad. As the condition improves, heat, massage, and corrective exercises are indicated. As soon as the muscle spasm has subsided, the patient is turned on his abdomen and moist heat applied to the back. A moist towel covers the back while reflected heat is used for about 30 minutes. 
On the case becoming ambulatory, external supports, either strapping or a back brace, are used for the lower back. If there is still deformity, it is corrected in an upright extension frame and the correction maintained by means of a plaster-of-paris cast. This is worn for a period of several weeks and the patient may or may not be up and about with crutches, depending upon the tolerance for weight-bearing. Whenever pain is referred to one lower extremity, a part or all of that member may be included in the cast. Where the pain is due to a postural fault, the routine is to correct the static error with a brace or corset and start a course of physio-therapy, consisting of corrective exercises with deep massage and exercises to tone the weakened musculature. Other static faults, such as a weak foot or such skeletal pathological conditions as hip disease and so forth, have to be considered in the care of the patient as they frequently play an important rôle.

Manipulative treatment under anæsthesia is given to those resistent cases of sprain which, after a reasonable amount of time, fail to respond to physiotherapeutic measures. 'This form of treatment is being used successfully more and more in low back cases of a chronic type. It is obviously the method of choice where there is any suggestion of the so-called subluxation of the sacro-iliac joint - usually a strain of the joint rather than malposition. It aims at breaking down adhesions and restoring mobility, and, where the condition is a chronic one associated with stiffness and limitation of motion, and there is no contraindication, it is a reasonable way to start the treatment.

A systematic routine is employed in carrying out the manipulation and the routine here described deals with the lower spinal, sacro-iliac, lumbo-sacral, and to some extent with the hip joints. One leg, with the knee straight, is flexed on the abdomen to its fullest extent, while the other one is hyperextended. A similar manœuvre is then carried out with the other leg. Both legs, with knees extended, are then flexed together on to the abdomen and this movement is continued until flexion is produced at the lumbo-sacral region. The spine is now rotated: the operator stands first on the right side of the patient while his assistant, on the other side of the operating table, steadies the patient's left shoulder and right thigh on the table. The operator now takes the patient's left thigh and pulls it towards him, so rotating the spine. The maximum rotation is produced by the operator's left forearm pulling the gluteal region of the patient towards him. The same is next done with the other leg. The patient is now turned on his face and first one leg and then the other hyper-extended at the hip joint, and then both legs are hyper-extended and extension of the spine produced. Often one hears during these manouvres loud, cracking sounds. One presumes that this is the replacement of a relaxed or subluxated sacro-iliac joint, or much more commonly the rupturing of some adhesions.

Operative treatment is reserved for those inveterate cases which fail to respond to all efforts by conservative means. Before operation is considered the utmost care must be taken to localize accurately the site of the affection. Fusion is carried out in the case of a sacro-iliac joint after the manner of Smith-Petersen; in the lumbo-sacral joint, an Albee's arthrodesing operation is used. 